К. Ю. Дергачёв, Л. А. Краснов, А. А. Челядин

Национальный аэрокосмический университет “ХАИ”, Харьков, Украина

\title{
ПОВЫШЕНИЕ ЭФФЕКТИВНОСТИ РАБОТЫ АЛГОРИТМОВ ДЕТЕКТИРОВАНИЯ ЛИЦ ПО МЕТОДУ ВИОЛЫ-ДЖОНСА
}

\begin{abstract}
Предложены новые методы повышения эффективности работы алгоритмов обнаружения и детектирования лиц на цифровых изображениях и видеопоследовательностях, основанных на методе Виолы-Джонса, и используемых при решении задач распознавания лиц. Это позволяет устранить влияние одного из главных факторов помех - компенсировать влияние изменений уровня освещенности сцены на качество обнаружения лиц. Для этого в классическую структуру этих алгоритмов дополнительно введена процедура автоматической стабилизации яркости кадра. Описана структура алгоритмов, для них разработано программное обеспечение на языке Python с использованием ресурсов библиотеки OpenCV, что позволяет вести обработку видеоданных в реальном масштабе времени. Предложена и программно реализована оригинальная методика оценки эффективности работы алгоритма по критерию максимума вероятности правильного обнаружения лиц и их главных элементов (глаз, носа, рта). Сопоставляются результаты работы классических и предложенных алгоритмов. Приводятся примеры работы и результаты тестирования программного обеспечения.
\end{abstract}

Ключевые слова: обнаружение и детектирование лиц, метод Виолы-Джонса, автоматическая стабилизация яркости кадра, вероятность правильного обнаружения лиц и их главных элементов.

\section{Введение}

Обнаружение лиц на изображениях является важнейшим предварительным этапом при решении задач более высокого уровня - распознавания лица, деталей лица или его мимики. Результаты надежного и достоверного решения задачи обнаружения позволяют получить информацию о присутствии и количестве лиц на изображении. Обычно такую информацию принято использовать в системах автоматического учета числа посетителей; системах пропускного контроля в учреждениях, аэропортах и метро; автоматических системах предотвращения несчастных случаев и пр. Отметим, что практические потребности в повышении качества распознавания лиц в самых разнообразных приложениях постоянно возрастают. А это требует неуклонного повышения качества обработки видеоданных и совершенствования алгоритмов распознавания лиц в сложных условиях.

Рассмотрим более подробно решение первой части общей задачи - обнаружения и детектирования лиц. Сейчас наиболее популярным методом для поиска области лица на изображении является метод Виолы-Джонса из-за его высокой скорости и эффективности $[1,2]$. Сущность этого метода основана на интегральном представлении изображения, методе построения классификаторов на базе алгоритма адаптивного бустинга (AdaBoost), и методе комбинирования классификаторов в каскадную структуру. В методике Виолы-Джонса впервые использованы каскады вейвлетов Хаара, обеспечивающие разбивку заданной прямоугольной области на разнотипные прямоугольные подобласти. Это позволяет построить детектор лиц, работающий в режиме реального времени с довольно высоким качеством. Однако существует ряд возмущающих факторов, ограничивающих эффективность работы алгоритма. Главный из них - это плохое качество освещения сцены.

Цель работы - создание алгоритмов детектирования лиц, обеспечивающих автоматичес-кую стабилизацию яркости анализируемого изображения независимо от уровня освещенности и методики оценки эффективности их работы в сравнении с классическим алгоритмом.

\section{Методы и средства построения новых алгоритмов}

Рассмотрим основные методы и средства для решения поставленной задачи.

Используемые ресурсы. При создании алгоритмов авторы использовали язык программирования Python и ресурсы библиотеки OpenCV [3, 4]. Этот выбор обусловлен открытым доступом к программным продуктам и их совместимостью с операционными системами Windows, Linux и Android. Работа алгоритмов также достаточно легко реализуется и на одноплатном компьютере Raspberry Pi.

Используемый набор средств программирования не оказывает большой нагрузки на процессор. Для существенного увеличения быстродействия при обработке видеоданных все основные процедуры реализованы с помощью стандартных функций OpenCV.

Стабилизация контрастности видеоданных. Обнаружение и регистрация лиц с помощью различных видеорегистраторов обычно происходит на фоне различного рода помех. Одним из доминирующих негативных факторов является изменчивость освещенности сцены. Это могут быть как быстро меняющиеся условия освещения, так и медленные его изменения, обусловленные например наступлением сумерек. Все это приводит к плохо контролируемым вариациям контрастности кадров, а значит и к ухудшению качества обработки. Для преодоления этих трудностей (неконтролируемых изменений контрастности кадров в зависимости от уровня освещенности) предложено исходную видеопоследовательность из цветового пространства $R G B$ конвертировать в пространство $Y U V$ с помощью функции: 
img yuv $=\mathrm{cv} 2 . \mathrm{cvtColor}$

(img, cv2.COLOR_BGR2YUV).

Обычно диапазон значений $R G B$ составляет [0 $\div 255]$ для каждой компоненты, а для цветового пространства $Y U V$ используются диапазоны:

- $Y \rightarrow[0 \div 255]$;

- $U \rightarrow[-112 \div 112]$

- $V \rightarrow[-157 \div 157]$.

В цветовом пространстве $Y U V$ [5] используется явное разделение информации о яркости и цвете. Цвет представляется в виде трех компонент - яркостной $(Y)$ и двух цветоразностных $(U$ и $V)$.

Поэтому после перевода кадра $R G B$ видеопоследовательности в цветовое пространство $Y U V$ в нем осуществляется процедура эквализации (повышения контрастности) только для компоненты $Y$ с помощью функции:

$$
\begin{aligned}
& \text { img_yuv }[:,:, 0]= \\
& \text { cv2.equalizeHist (img_yuv }[:,:, 0]),
\end{aligned}
$$

а затем производится обратное преобразование кадра из формата $Y U V$ в формат $R G B$ :

img_output=cv2.cvtColor

(img_yuv, cv2.COLOR_YUV2BGR).

При этом баланс цвета сохраняется без изменений, так как цветоразностные компоненты $U$ и $V$ преобразованиям не подвергались.

Переход из цветового пространства $R G B$ в пространство $Y U V$ позволяет просто оценить средний уровень яркости кадра по компоненте $Y$. Наиболее объективным и устойчивым показателем на наш взгляд является $M B$ (Medium Brightness), который вычисляется как

$$
M B=\frac{1}{M N} \sum_{i=0}^{N} \sum_{j=0}^{M} Y(i, j)
$$

где $Y(i, j)$ - двумерный массив чисел, определяю-
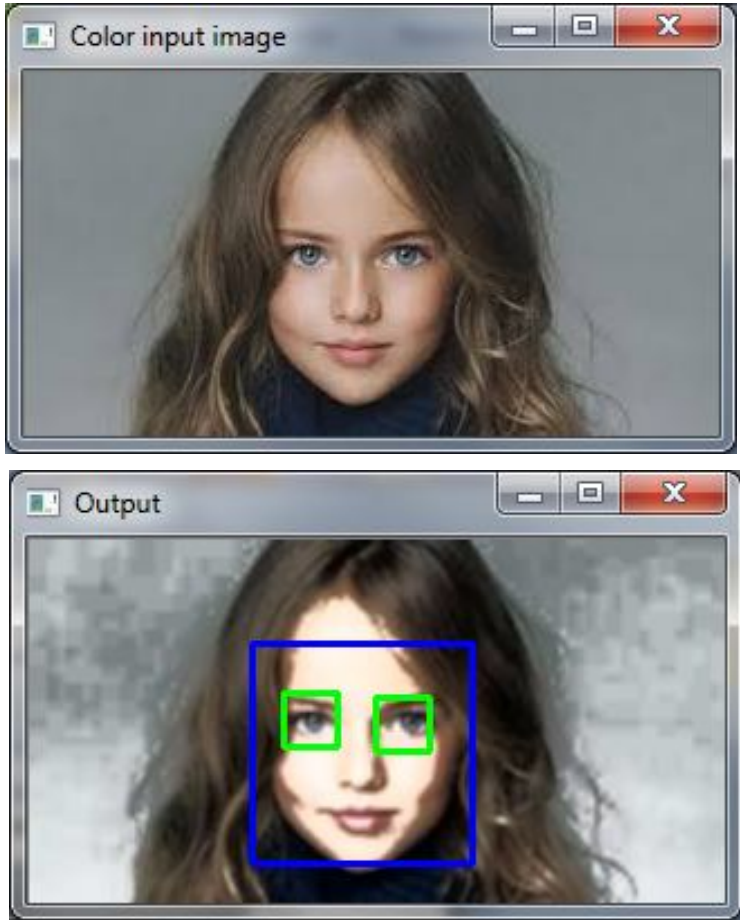

Рис. 1. Обнаружение лица и глаз с использованием процедуры эквализации при слабой освещенности сцены щих яркость пикселей изображения кадра размером $M \times N$. Этот показатель тем более полезен, что при малых уровнях яркости кадра в системах видеонаблюдения для распознавания лиц удобно использовать процедуру его сравнения с предварительно установленным порогом для автоматического включения/выключения системы подсветки сцены. Однако, использование показателя средней яркости $M B$ вызывает и существенные затруднения. В библиотеке OpenCV отсутствует оптимизированная функция вычисления нормированной средней яркости изображений, а циклическое суммирование показателей яркости пикселей по всему кадру (в особенности больших размеров) слишком замедляет работу алгоритмов и не дает вести обработку в реальном масштабе времени. Поэтому в предлагаемом алгоритме этот показатель не используется, а контроль уровня освещенности сцены ведется адаптивно на основании иных показателей, работа которых будет описана далее.

Эквализация обеспечивает выравнивание гистограмм и приводит показатель средней яркости изображения к значению ( $M B=127)$ независимо от того, каким этот показатель был для исходного изображения. Стабилизация уровня средней яркости кадров упрощает обработку видеоданных. Это делает алгоритм более устойчивым к воздействию внешних факторов. Использование процедуры эквализации в работе алгоритма обнаружения лица и глаз показано на рис. 1.

Обнаружение и детектирование лиц методом Виолы-Джсонса. Большинство современных систем распознавания при поиске и выделении лиц ориентированы на использование метода ВиолыДжонса. Он основан на интегральном представлении изображения, построении классификаторов на базе алгоритма адаптивного бустинга и создании классификаторов в виде каскадной структуры.
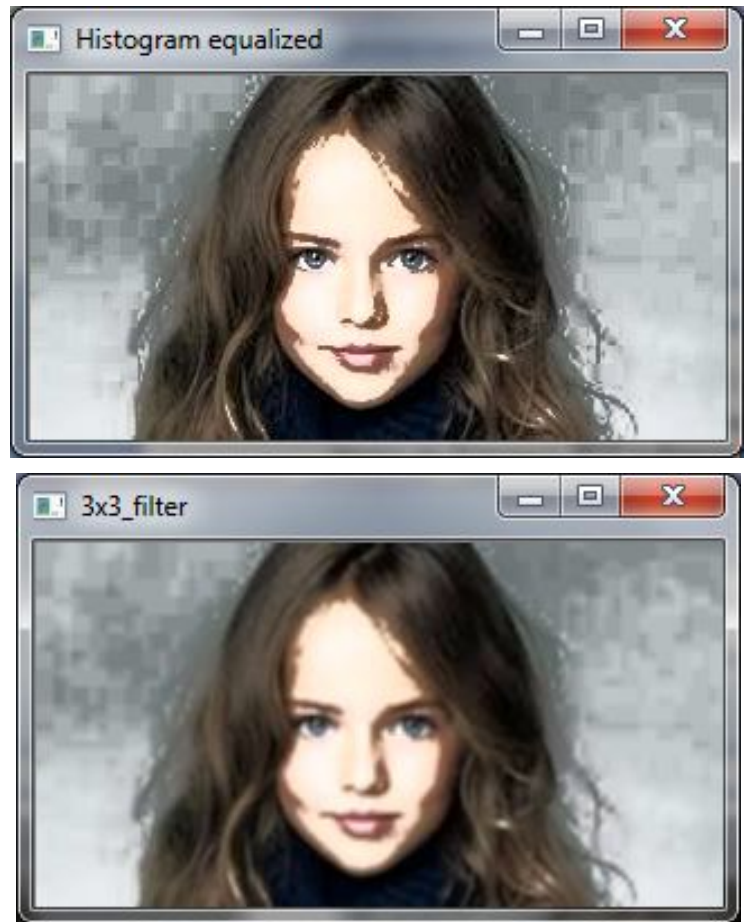
Наиболее трудоемким является процесс обучения каскадов Хаара с помощью алгоритма машинного обучения AdaBoost. Однако, в настоящее время для каскадных классификаторов Хаара существует большое количество уже обученных каскадов, в том числе в стандартной поставке библиотеки OpenCV. Её установочный пакет содержит целый набор готовых обученных классификаторов, сохраненных в виде файлов с расширением «* . xml». В этом наборе имеются классификаторы, как для поиска лица, так и его отдельных частей (глаз, рта, носа).

Не останавливаясь подробно на предназначении и свойствах отдельных классификаторов, отметим, что особый интерес среди них представляют те, которые позволяют определить положение глаз. Это очень полезная процедура, поскольку она дает возможность оценки расстояний между зрачками, и при дальнейшем анализе позволяет устранять факторы, связанные с наклоном головы. Классификаторы, обученные поиску глаз, весьма чувствительны к наличию очков. В большинстве случаев они дают сбой, особенно для очков полностью скрывающих глаза. В таких случаях следует использовать более эффективный классификатор для обнаружения глаз:

haarcascade_eye_tree_eyeglasses.xml»

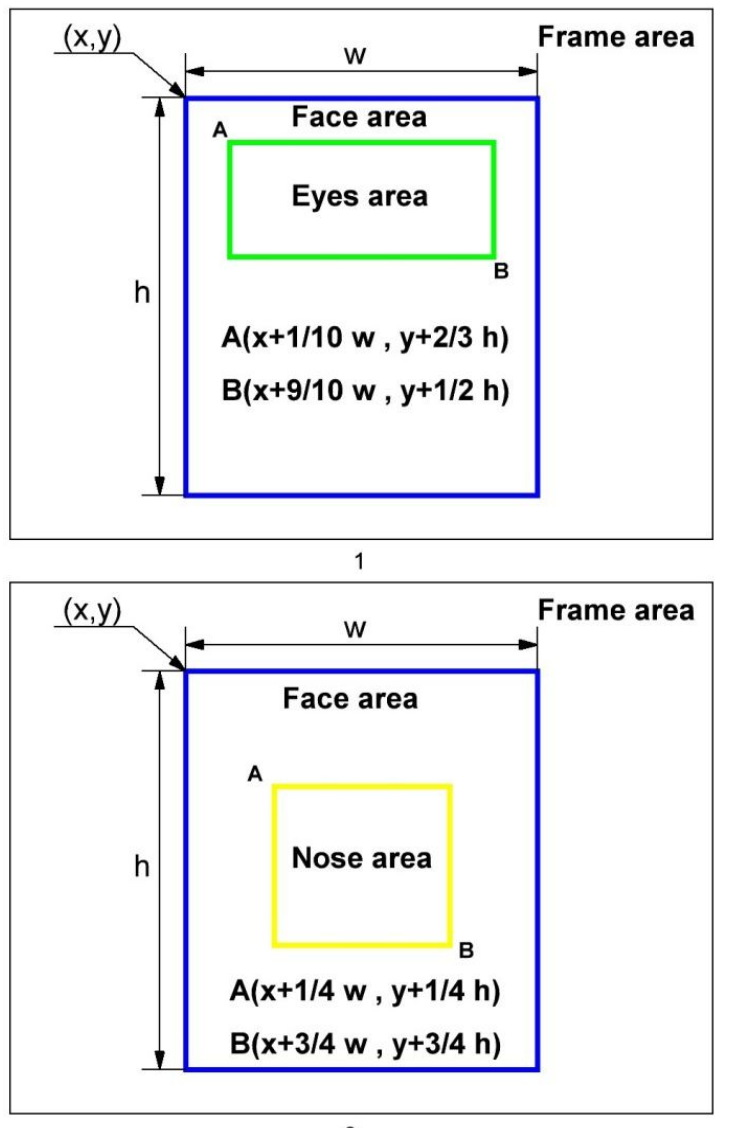

Он обучен поиску на изображении глаз в очках, и может быть использован как запасной вариант в случаях сбоя в работе обычного классификатора.

Наиболее эффективными являются методы поиска основных элементов в уже выделенной области лица, так как это локализует регион поиска и сокращает время анализа, а также значительно понижает вероятность ложных срабатываний. Выбор областей интереса для обнаружения глаз, носа и рта требует оптимизации положения и размеров области поиска для каждого элемента лица. Примеры такого разбиения выделенной области на зоны поиска отдельных элементов лица в предлагаемом алгоритме показан на рис. 2. Размеры областей поиска для нашего алгоритма определялись экспериментально, но при необходимости могут быть оптимизированы дополнительно. Обратите внимание, что существует две возможности обнаружения глаз - выделение общей для двух глаз области интереса (или области поиска) и поиск и обнаружение левого и правого глаза отдельно. В библиотеке OpenCV для этого предусмотрены соответствующие классификаторы. Какой из этих методов предпочтительнее, в нашей работе будет определено экспериментальным путем.
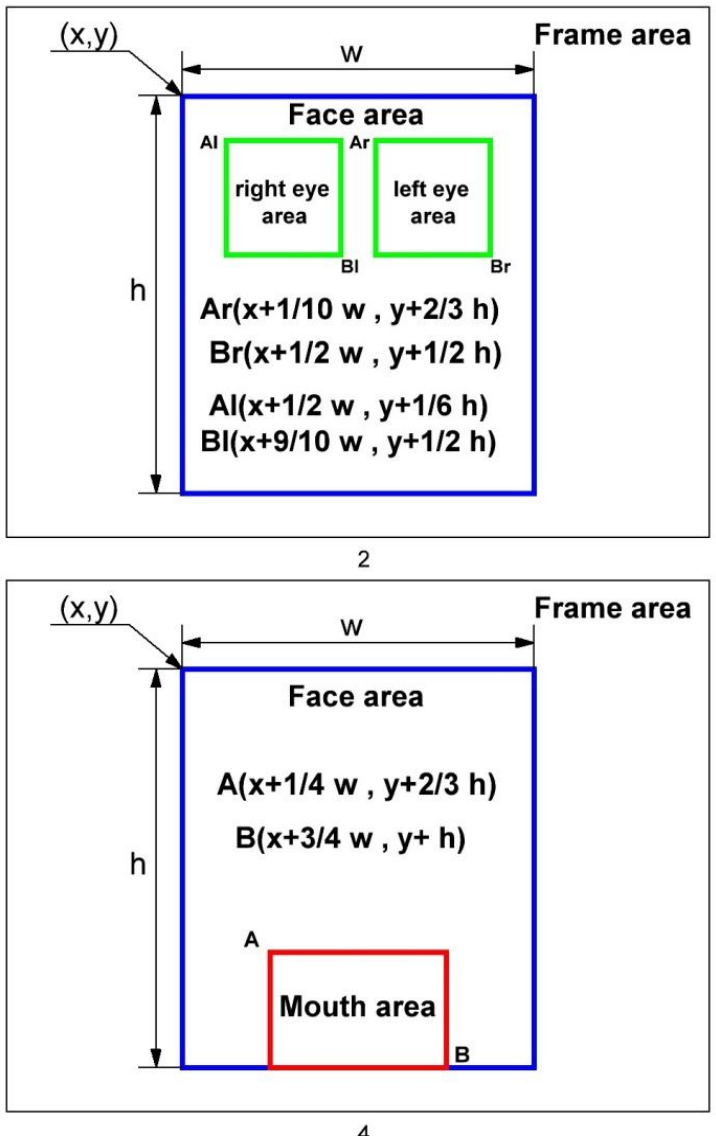

Рис. 2. Зоны поиска лиц и их элементов в кадре (координаты отдельных зон поиска)

Показатели качества работы алгоритмов и методы их использования. Объективным критерием эффективности работы любого алгоритма детектирования лиц и их элементов в кадрах видеопоследовательности является показатель вероятности правиль- ного обнаружения лица при условии его наличия в кадре. Процедура обнаружения лица в текущем кадре с применением соответствующего каскадного классификатора в случае успеха завершается построением прямоугольика с помощью функции OpenCV 
cv2. rectangle (frame, $(\mathrm{x}, \mathrm{y})(\mathrm{x}+\mathrm{w}, \mathrm{y}+\mathrm{h})$, $255,0,0), 2)$.

Успешное завершение детектирования лица в программном коде алгоритма необходимо сопровождать появлением события логической 1; в противном случае - появлением логического 0.

Для создания устойчивого критерия качества правильного обнаружения лиц рассчитывалась вероятность $P n$, полученная по результатам подсчета в скользящем окне размером 100 кадров количества успешных обнаружений. При стандартной скорости смены кадров видеокамеры 30/с окно имеет постоянную времени изменения $P n$ примерно равную 3,3 с. Качество работы детектора можно считать удовлетворительным при $P n \geq 0,9$. По показателю вероятности правильного обнаружения лиц в кадре легко анализировать влияние различных факторов (в том числе изменений освещенности, геометрических факторов и пр.) на качество работы алгоритмов обнаружения и детектирования лиц.

Аналогичным образом рассчитываются и вероятности правильного обнаружения глаз, носа, рта в соответствующих областях. Эти показатели вычисляются как условная вероятность такого события при условии правильного обнаружения всего лица. С достоверностью приемлемой для практического использования можно считать события обнаружения отдельных элементов лица независимыми. Следовательно, вероятность правильного обнаружения всех элементов можно оценивать как произведение вероятностей обнаружения этих элементов.

Для адаптации предлагаемых в нашей работе алгоритмов детектирования лиц к условиям изменения внешнего освещения сцены предложено организовать два канала обработки видеоданных (схема на рис. 3). В одном из них исходный видеопоток используется непосредственно для обнаружения лиц, а во втором канале производится его предварительная эквализация для приведения яркости кадра к среднему значению. На выходах обоих каналов обработки в скользящих окнах размером в 100 кадров вычисляются вероятности правильного обнаружения лиц и их элементов, а затем их сопоставляют в блоке сравнения. $\mathrm{B}$ зависимости от знака разницы вероятностей $\Delta P n \mathrm{c}$ помощью петли обратной связи на выход системы передаются результаты обнаружения лиц из того канала, в котором условия освещения более комфортны для работы алгоритма обнаружения лиц.

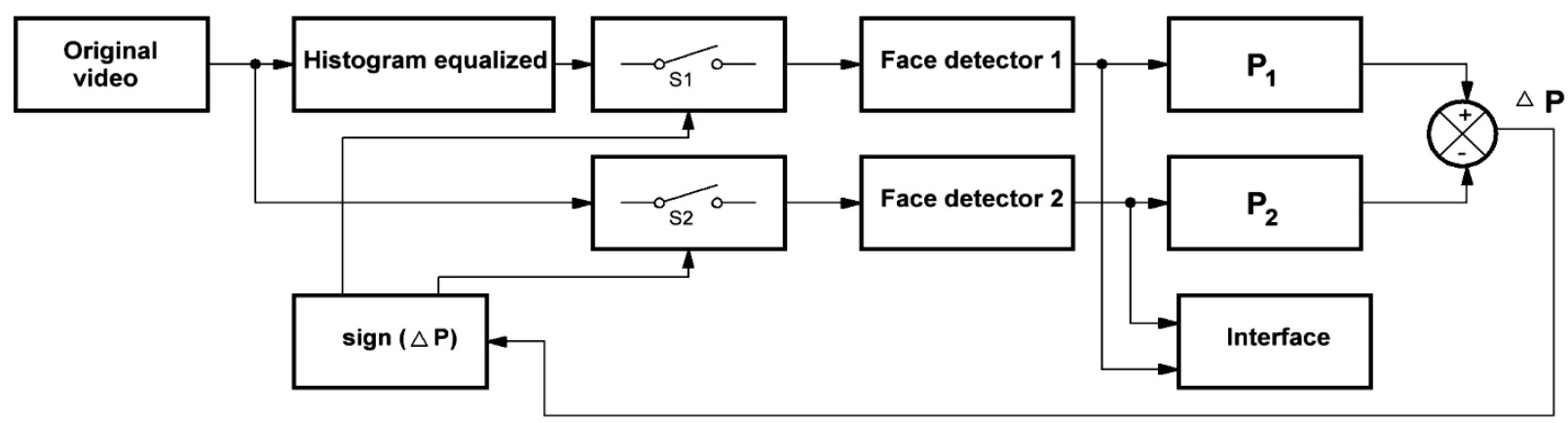

Рис. 3. Обобщенная структура адаптивного алгоритма детектирования лиц

По мнению авторов, такой метод адаптации к изменениям освещенности сцены существенно лучше метода оценки показателя средней яркости кадра $M B$, так как его использование не накладывает существенных ограничений на скорость обработки видеоданных.

\section{Практическая реализация алгоритмов}

Рабочие версии алгоритмов обнаружения лиц создавались с учётом описанных выше подходов и ресурсов. Была поставлена задача обнаружения лиц, глаз, носа и рта. При этом обнаружение глаз осуществлялось двумя способами - с использованием классификатора для детектирования глаз в общей зоне поиска и классификаторов раздельного обнаружения (правого и левого глаза отдельно). Далее сравним эти два способа по показателю вероятности обнаружения глаз. Для экономии времени, ресурсов и простоты реализации алгоритмов в нашем проекте использовался набор предварительно обученных каскадных классификаторов Хаара для соответствующих элементов лица, импортированный из библиотеки OpenCV. Считаем уместным напомнить о необходимости размещения этих классификаторов в корневой папке Python. Это поможет избежать ошибок при поиске путей обращения к ним и ускорить обработку исходных данных. Далее приведен перечень использованных классификаторов:

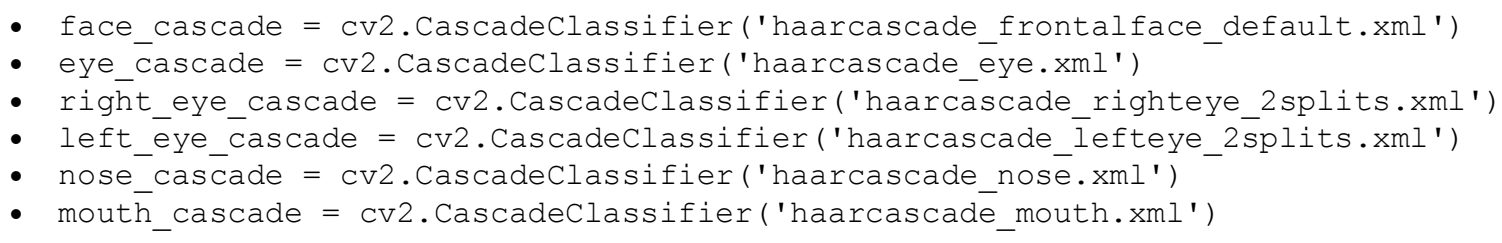

Результаты обнаружения одного или нескольких лиц с помощью предложенных алгоритмов показаны на рис. 4. Следует отметить, что наиболее устойчивые результаты обнаружения лица и его элементов наблю- даются при условии фронтально расположенного по отношению к лицу источника освещения сцены, Благоприятный геометрический фактор процедуры обнаружения предполагает наклоны головы не более $30^{\circ}$. 

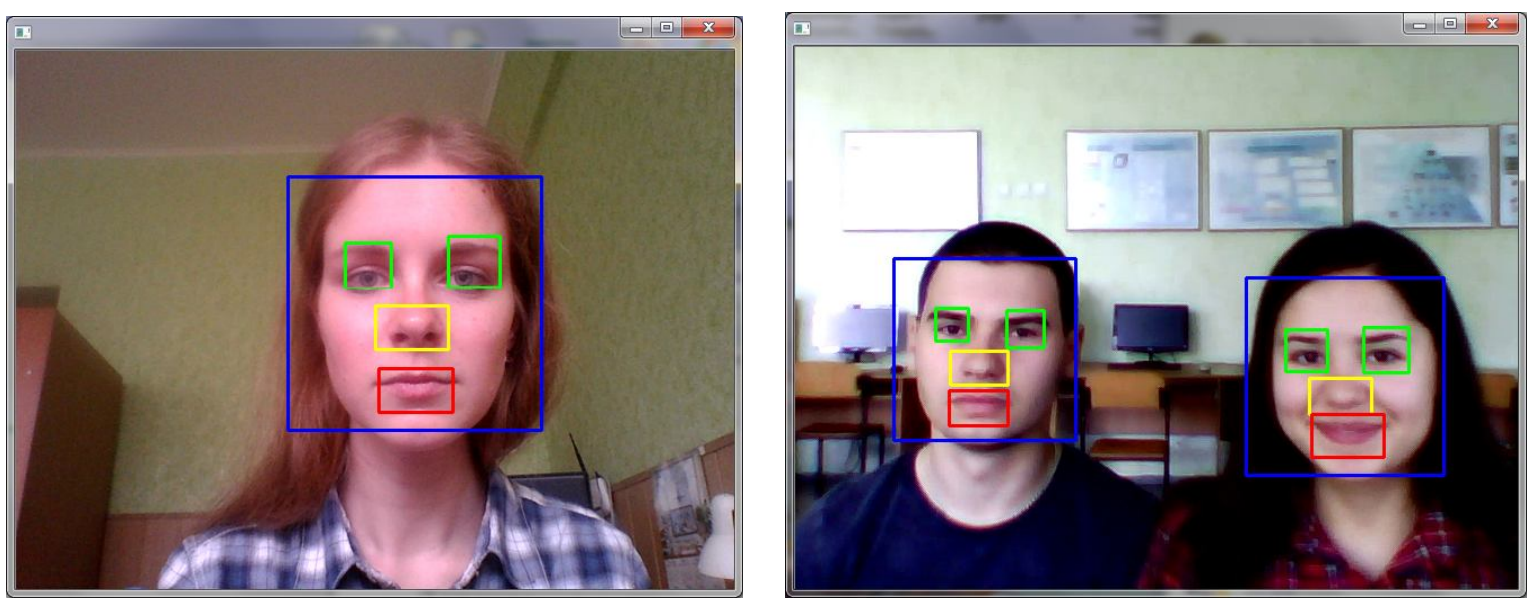

Рис. 4. Результаты обнаружения лиц и их элементов в кадре

Поскольку методы и средства создания алгоритмов уже обсуждались достаточно подробно, далее остановимся только на структурных особенностях построения новых алгоритмов. Рассмотрим два различных варианта построения адаптивных алгоритмов обнаружения лиц и их фрагментов на видеопоследовательностях и оценим преимущества и недостатки каждого из них. Структурные схемы этих алгоритмов приведены на рис. 5 и 6 . В первом вари- анте полностью реализован принцип адаптации к изменениям уровня освещенности сцены, основанный на определении значений текущей разницы вероятностей правильного обнаружения лиц и их элементов при использовании процедуры эквализации кадра и без неё (рис. 6). Основным недостатком такого метода является заметное уменьшение быстродействия, поскольку в этой ситуации объем вычислительных операций фактически удваивается.

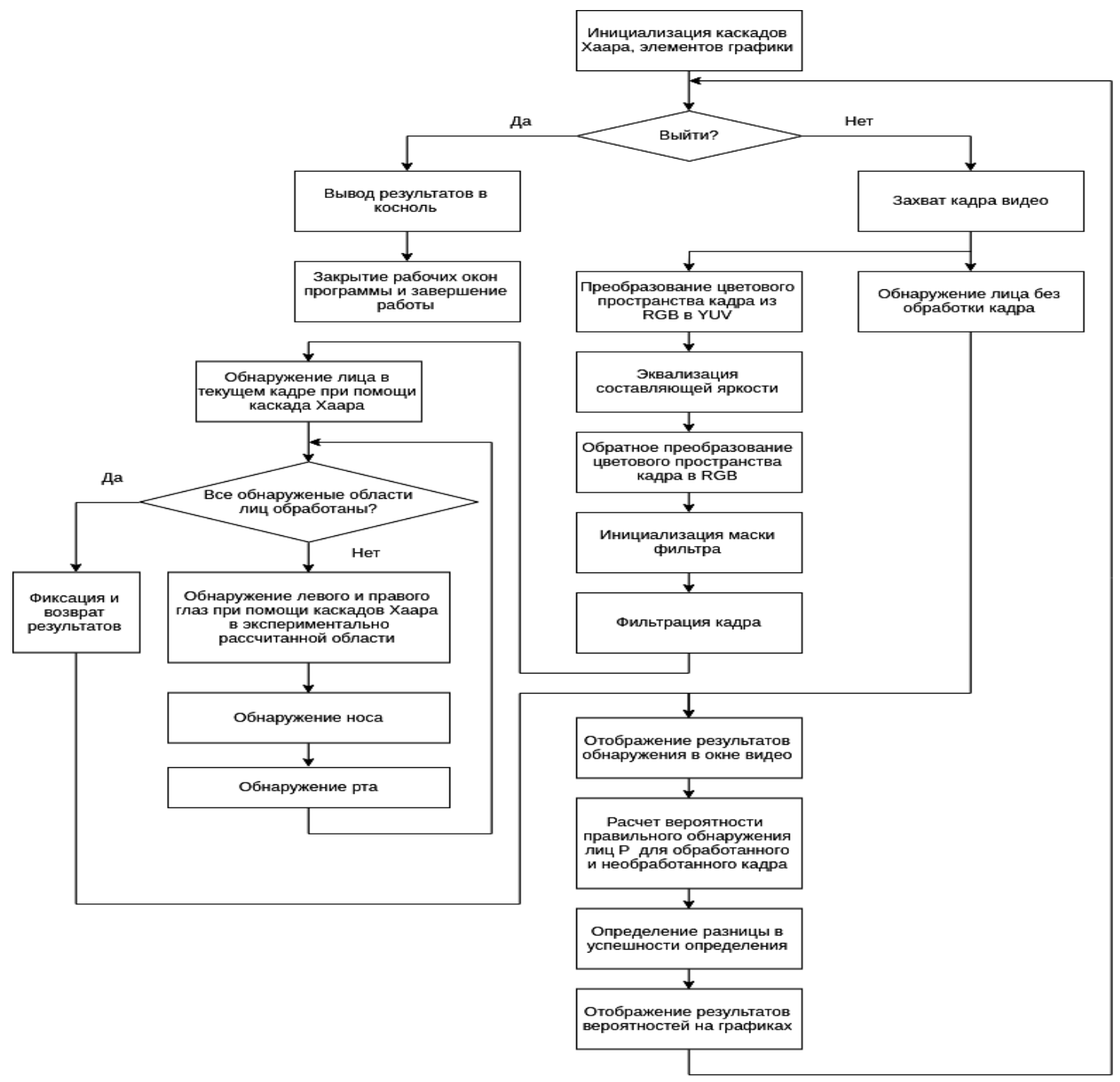

Рис. 5. Адаптация алгоритма к условиям освещения по вероятности правильного обнаружения лиц в кадре 


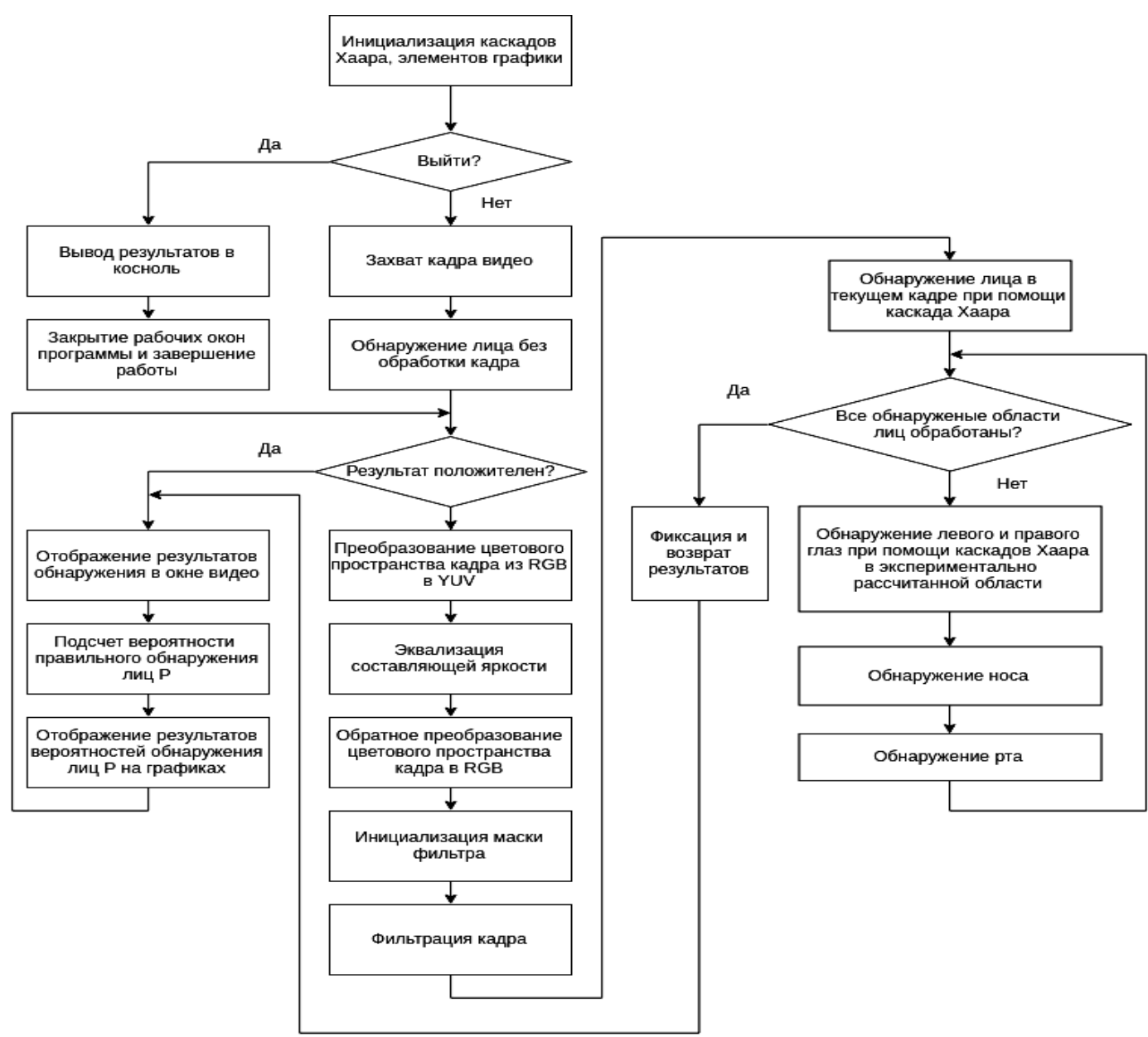

Рис. 6. Упрощенный вариант адаптации алгоритма обнаружения лиц к условиям освещения

Представленный на рис. 6 вариант построения адаптивного алгоритма поиска лиц в кадрах видео существенно проще, поскольку процедура эквализации видеоданных используется только в тех ситуациях, когда из-за плохих условий освещения в текущем кадре обнаружение лица не состоялось, несмотря на его присутствие в кадре. Такой подход существенно экономичнее - количество вычислений практически вдвое меньше чем в первом варианте построения алгоритма. Это позволяет уверенно осуществлять обработку видеоданных в реальном масштабе времени.

\section{Результаты экспериментальных исследований}

Специфические свойства алгоритмов обнаружения лиц, основанных на использовании обученных каскадных классификаторов, определяют вероятностный характер их функционирования. Поэтому основным показателем качества таких алгоритмов в нашей работе принято считать вероятность правильного обнаружения лица и его элементов. Для наглядности получаемых результатов в первом варианте построения алгоритма обнаружения была синтезирована специальная графическая форма, внутри которой в отдельных окнах отслеживаются в динамике вероятности обнаружения лиц при работе без применения процедуры эквализации (красные кривые) и с её применением (синие кривые) для лица и его элементов (глаз, носа и рта). Кроме этого, в левом ниж- нем окне данной формы отображается текущая разность вероятностей правильного обнаружения (при эквализации кадра видео и без неё). Вид этой обобщенной отчетной формы наглядно показан на рис. 7, а. Аналогичным образом была построена графическая форма для текущей оценки эффективности обнаружения и для второго алгоритма. Она содержит четыре окна, в которых отображаются текущие значения вероятностей правильного обнаружения лица, глаз, носа и рта (рис. 7, б). Эти средства визуализации текущих процессов обнаружения достаточно эффективны, но они не дают ответа на основной вопрос какова эффективность работы алгоритма при длительном анализе видеопоследовательности?

Для обобщенной оценки эффективности работы алгоритмов возможны два метода - качественный (визуальный) и количественный. В первом случае проводится визуальное наблюдение за разноцветными прямоугольниками, ограничивающими обнаруженное лицо и его элементы (глаза, нос и рот). Если процесс их отрисовки воспринимается как непрерывный, то качество детектирования можно считать приемлемым (равным 100 \%). Однако, инерционность зрения наблюдателя при высокой частоте смены кадров (30/c) не позволяет визуально воспринять пропуски обнаружения отдельных кадров. Это может существенно исказить результаты тестирования. Поэтому для количественной оценки эффективности в программе для всех кадров видео- 
последовательности формируется одномерный массив (1 - по факту обнаружения лица в кадре, и 0 - в случае пропуска лица), по которому строятся вероятностные характеристики качества работы алгоритма. Однако, следует иметь ввиду, что для этого необходима достоверно аннотированная тестовая видеопоследовательность. Простейший вариант такой последовательности - видеоряд, у которого в каждом кадре присутствует лицо для обнаружения,

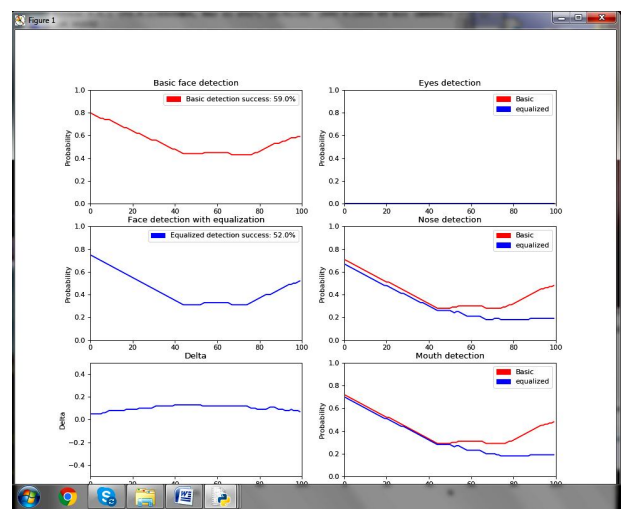

a соблюдены условия освещения (фронтальный источник света) и поддерживается геометрический фактор (наклон головы менее $30^{0}$ ). Разумеется, можно использовать и более сложный тестовый видеоряд, у которого в определенные интервалы времени лица в кадре отсутствуют. Тогда легко оценить не только вероятности правильного обнаружения лиц, но и вероятности ложного обнаружения (обнаружения лица при условии его отсутствия в кадре).

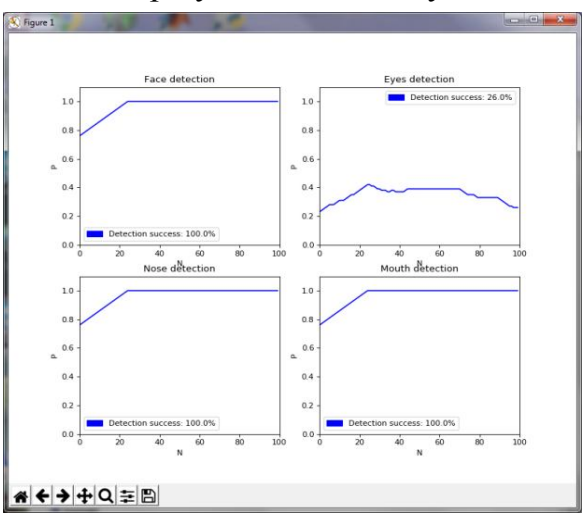

б

Рис. 7. Окна текущего контроля вероятностей правильного обнаружения лиц и их элементов

На рис. 8 показана форма обобщенной оценки эффективности работы алгоритма обнаружения лиц и их главных элементов для видеоряда продолжительностью 1000 кадров. Длительность такой записи составляет $\sim 33$ с. Для процедур обнаружения всех элементов построены периодограммы, где для каждого из $\mathrm{N}$ кадров в соответствие ставится 1 (при успешном обнаружении) и 0 (при необнаружении) соответствующего элемента лица. Кроме этого, для каждой периодограммы вычисляется вероятность правильного обнаружения на выборке из 1000 кадров. Такая форма позволяет получить не только обобщенные оценки вероятностей обнаружения, но и наглядно показывает, на каких кадрах обнаружение не было выполнено.

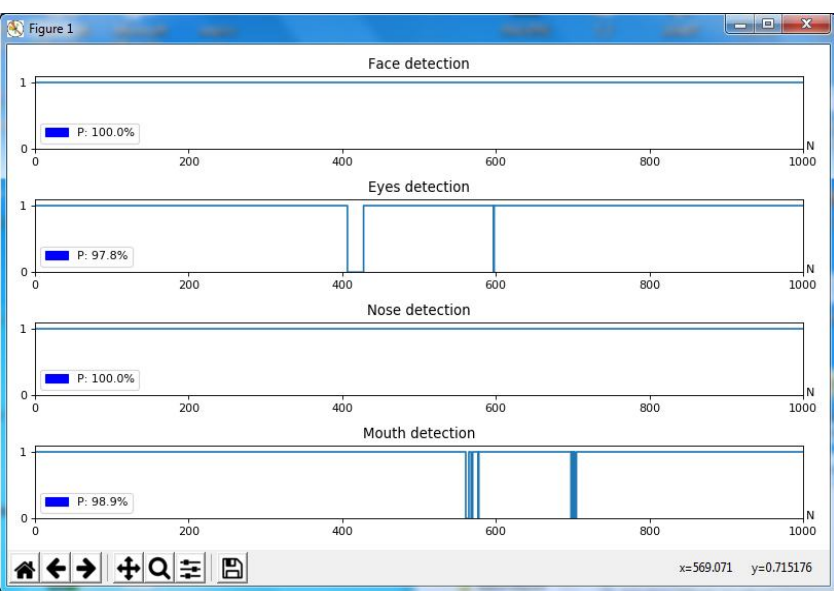

Рис. 8. Окно оценки эффективности обнаружения лица и его главных элементов по видеоданным

Для тестирования был сделан ряд видеозаписей, отвечающих заданным требованиям. Они выполнялись в формате *avi с размером кадров $480 \times 640$ пикселей с неподвижно установленной камеры. Артефакты, обусловленные движением человека в кадре, были исключены. Расстояние от лица до объектива видеокамеры составляло 1м. Для контроля уровня освещенности сцены использовался люксометр Ю-16 с фотоэлементом Ф-102, который позволяет фиксировать уровень освещенности с точностью $\pm 10 \%$.

Поскольку предложенные алгоритмы должны обеспечивать автоматическую стабилизацию яркости кадров анализируемой видеопоследовательности независимо от уровня освещенности, в первую очередь сопоставлялись результаты обнаружения лиц в записях с различными уровнями освещения сцены. Результаты этого эксперимента сведены в табл 1.

\section{Таблица 1 - Показатели эффективности} предлагаемых алгоритмов

Without equalization

\begin{tabular}{|c|c|c|c|c|}
\hline $\begin{array}{c}\text { Scene } \\
\text { illumination, } \\
\text { Lx }\end{array}$ & $\begin{array}{c}\text { Face de- } \\
\text { tection, } \\
\mathrm{P} \%\end{array}$ & $\begin{array}{c}\text { Eyes detec- } \\
\text { tion, } \\
\mathrm{P} \%\end{array}$ & $\begin{array}{c}\text { Nose detec- } \\
\text { tion, } \\
\mathrm{P} \%\end{array}$ & $\begin{array}{c}\text { Mouth } \\
\text { detection, } \\
\mathrm{P} \%\end{array}$ \\
\hline 100 & 100 & 96,5 & 98,1 & 97,2 \\
\hline 150 & 100 & 97,2 & 98,5 & 97,5 \\
\hline 200 & 100 & 97,8 & 98,7 & 97,7 \\
\hline \multicolumn{5}{|c|}{ With equalization } \\
\hline $\begin{array}{c}\text { Scene } \\
\text { illumination, }\end{array}$ & $\begin{array}{c}\text { Face de- } \\
\text { tection, } \\
\text { Lx }\end{array}$ & $\begin{array}{c}\text { Eyes detec- } \\
\text { tion, } \\
\text { P } \%\end{array}$ & $\begin{array}{c}\text { Nose detec- } \\
\text { tion, } \\
\mathrm{P} \%\end{array}$ & $\begin{array}{c}\text { Mouth } \\
\text { detection, } \\
\mathrm{P} \%\end{array}$ \\
\hline 100 & 100 & 97,1 & 98,6 & 97,4 \\
\hline 150 & 100 & 97,2 & 98,6 & 97,45 \\
\hline 200 & 100 & 97,1 & 98,62 & 97,4 \\
\hline
\end{tabular}

Анализ этих данных показал, что вероятность правильного обнаружения отдельных элементов лица возрастает по мере увеличения уровня освещенности при условии, что обработка данных проводится без автоматической регулировки средней яркости кадра. В случаях же, когда используется процедура эквализации для регулировки яркости, вероятность обнару- 
жения остается стабильной. Значит, при низком уровне освещенности эффективность алгоритмов с автоматической регулировкой яркости выше, а при ярком освещении сцены качество работы обоих типов алгоритмов примерно одинакова. Отметим высокое качество работы предложенных алгоритмов, поскольку похожие современные технические решения обеспечивают вероятность правильного обнаружения на уровне $95-97 \%$.

Кроме этого в работе было проведено исследование эффективности работы алгоритмов в зависимости от расстояния лица до видеорегистратора. Исходное расстояние составило 0,7 м, а конечное расстояние - 2,5 м. Остальные условия съемки были аналогичны предыдущим исследованиям. Отметим, что процедура обнаружения лица оказалась нечувствительной к увеличению расстояния между лицом и видеокамерой. Но при увеличении этого расстояния более чем 1,5 м это приводит к прерыванию процеду- ры обнаружения элементов лица (глаз, носа и рта). Это ограничение связано с относительным уменьшением размеров лица в кадре, и может быть устранено путем адаптивного управления его размерами.

\section{Заключение}

Предложены новые алгоритмы обнаружения и детектирования лиц на цифровых изображениях и видеопоследовательностях. В них реализована возможность адаптивно регулировать уровень средней яркости кадра, что позволяет улучшить основные показатели работы. Вероятность правильного обнаружения лиц для таких алгоритмов составляет $96 \%$. Они реализованы на языке Python с использованием ресурсов библиотеки OpenCV. Это позволило вести обработку данных в реальном масштабе времени. Использование этих результатов дает возможность повысить качество работы и достоверность результатов при распознавании лиц в различных системах.

\title{
REFERENCES
}

1. Viola P., Jones M.J. Rapid object detection using a boosted cascade of simple features // IEEE Conf. on Computer Vision and Pattern Recognition. - Kauai, Hawaii, USA, 2001. - V. 1. - P. 511-518.

2. Viola P., Jones M.J. Robust real_time face detection // Int. Journal of Computer Vision. - 2004. - V. 57. - № 2. - P. $137-154$.

3. Joseph Howse, Joe Minichino, Learning OpenCV 3 Computer Vision with Python, Packt Publishing, 2015, Packt Publishing.

4. Saurabh Kapur, Computer Vision with Python 3, Packt Publishing, August 2017, ISBN: 978-1-78829-976-3.

5. Prateek Joshi, OpenCV with Python By Example, Packt Publishing, September 2015, ISBN: 978-1-78528-393-2..

6. Библиотека компьютерного зрения OpenCV [Электронный ресурс]. - Режим доступа: http://docs.opencv.org/trunk/doc/py tututoria/py objdetect/py face detection/py face detection.html.

7. Разработка мультимедийных приложений с использованием библиотек OpenCV и IPP [Электронный ресурс] / А. В. Бовыкин [и др.]/ - Электрон. текстовые данные. - М.: Интернет-Университет Информационных Технологий (ИНТУИТ), 2016. - 515 с. - Режим доступа: http://www.iprbooksshop.ru/39564/

8. F. Comasch, S. Stuijk, T. Basten and H. Corporaal "Rasw: A runtime adaptive sliding window to improve viola-jones object detection", Distributed Smart Cameras (ICDSC), 2013.

Рецензент: д-р техн. наук, проф. О. В. Козелков, Державний університет телекомунікацій, Київ Received (Надійшла) 29.08.2018 Accepted for publication (Прийнята до друку) 22.09.2018

\section{Підвищення ефективності роботи алгоритмів детектування облич за методом Віоли-Джонса}

\author{
К. Ю. Дергачов, Л. О. Краснов, О. О. Челядін
}

Запропоновано нові методи підвищення ефективності роботи алгоритмів виявлення і детектування облич на цифрових зображеннях і відеопослідовність, заснованих на методі Віоли-Джонса, і використовуваних при вирішенні задач розпізнавання облич. Це дозволяє усунути вплив одного з головних чинників перешкод - компенсувати вплив змін рівня освітленості сцени на якість виявлення осіб. Для цього в класичну структуру цих алгоритмів додатково введена процедура автоматичної стабілізації яскравості кадру. Описано структуру алгоритмів, для них розроблено програмне забезпечення на мові Python з використанням ресурсів бібліотеки OpenCV, що дозволяє вести обробку відеоданих в реальному масштабі часу. Запропоновано і програмно реалізована оригінальна методика оцінки ефективності роботи алгоритму за критерієм максимуму ймовірності правильного виявлення облич і їх головних елементів (очей, носа, рота). Зіставляються результати роботи класичних і запропонованих алгоритмів. Наводяться приклади роботи та результати тестування програмного забезпечення.

Ключ ов і сл ов а : виявлення і детектування облич, метод Віоли-Джонса, автоматична стабілізація яскравості кадру, ймовірність правильного виявлення осіб і їх головних елементів.

\section{Increasing the effectiveness of the algorithms for detecting faces using the Viola-Jones method}

K. Dergachov, L. Krasnov, O. Cheliadin

There are proposed new methods for increasing the efficiency of algorithms for detecting faces on digital images and video sequences based on the Viola-Jones method and used in solving face recognition problems. This allows to eliminate the influence of the one main interference factors - to compensate the effect of changes in the scene illumination level on face detection quality. For this purpose, automatically stabilizing frame brightness procedure is additionally introduced into the classical structure of these algorithms. The structure of algorithms is described and software is developed using Python programming language and OpenCV library resources, that allows to conduct video data processing in real time. There is proposed and programmatically implemented an original method for estimating the algorithm efficiency based on the maximum probability criterion of correct faces and their main elements (eye, nose, mouth) detection. The results of the classical and proposed algorithms are compared. Examples of work and software testing results are given.

Keywords : face detection, Viola-Jones method, frame brightness automatic stabilization, face and its parts detection probability. 